\title{
OPEN Machine learning based prediction of lattice thermal conductivity for half-Heusler compounds using atomic information
}

\author{
Hidetoshi Miyazaki ${ }^{1,2}$, Tomoyuki Tamura $^{1}$, Masashi Mikami ${ }^{3}$, Kosuke Watanabe ${ }^{1,5}$, \\ Naoki Ide ${ }^{1}$, Osman Murat Ozkendir ${ }^{4}$ \& Yoichi Nishino ${ }^{1}$
}

Half-Heusler compound has drawn attention in a variety of fields as a candidate material for thermoelectric energy conversion and spintronics technology. When the half-Heusler compound is incorporated into the device, the control of high lattice thermal conductivity owing to high crystal symmetry is a challenge for the thermal manager of the device. The calculation for the prediction of lattice thermal conductivity is an important physical parameter for controlling the thermal management of the device. We examined whether lattice thermal conductivity prediction by machine learning was possible on the basis of only the atomic information of constituent elements for thermal conductivity calculated by the density functional theory in various half-Heusler compounds. Consequently, we constructed a machine learning model, which can predict the lattice thermal conductivity with high accuracy from the information of only atomic radius and atomic mass of each site in the half-Heusler type crystal structure. Applying our results, the lattice thermal conductivity for an unknown half-Heusler compound can be immediately predicted. In the future, low-cost and shorttime development of new functional materials can be realized, leading to breakthroughs in the search of novel functional materials.

Thermal conductivity is a physical quantity representing how heat is transferred from one side of a material to the other when thermal energy is applied. It is one of the most fundamental and important physical quantities. Moreover, it is an important physical quantity in terms of application, which is necessary for the understanding of thermal management to ensure the performance, life-time, and safety for thermoelectric energy conversion devices, and spintronics technology.

Thermal conductivity can be divided into electron and lattice contributions. The thermal conductivity of electrons can be determined from the electrical conductivity using the Wiedemann-Franz law. Half-Heusler compounds have a high lattice thermal conductivity due to their high crystal symmetry. Therefore, it is necessary to know the exact lattice thermal conductivity for thermal management in half-Heusler devices. Theoretical predictions of the lattice thermal conductivity of solids can be made using non-equilibrium molecular dynamics simulations $s^{1-7}$ or the density functional theory (DFT) calculations ${ }^{8-16}$. In recent years, with the advances in machine learning (ML) algorithms, several studies on understanding the heat transport properties of functional materials have been reported ${ }^{17-21}$. The prediction of thermal conductivity using non-equilibrium molecular dynamics requires an enormous amount of computational time because of the need to calculate the time evolution of the vast amount of atomic movements. On the other hand, because the DFT calculation can accurately calculate the interaction between atoms, the thermal conductivity can be predicted for several hundred times. Presently, theoretical studies of the lattice thermal conductivity are limited to systems with a small number of atoms in the unit cell, such as simple pure metals ${ }^{8,11,16}$, binary materials ${ }^{9,13-16,22}$, and full and half-Heusler

\footnotetext{
${ }^{1}$ Department of Physical Science and Engineering, Nagoya Institute of Technology, Nagoya 466-8555, Japan. ${ }^{2}$ Frontier Research Institute for Materials Science, Nagoya Institute of Technology, Gokiso-cho, Showa-ku, Nagoya 466-8555, Japan. ${ }^{3}$ National Institute of Advanced Industrial Science and Technology, 2266-98 Anagahora, Shimoshidami, Moriyama, Nagoya 463-8560, Japan. ${ }^{4}$ Department of Natural and Mathematical Sciences, Faculty of Engineering, Tarsus University, 33400 Tarsus, Turkey. ${ }^{5}$ Present address: Integrated Research Center for Energy and Environment, Kyushu Institute of Technology, 1-1 Sensui, Tobata-ku, Kitakyushu, Fukuoka 804-8550, Japan. ${ }^{\circledR}$ email: miyazaki@nitech.ac.jp
} 
(a) Prediction of lattice parameter

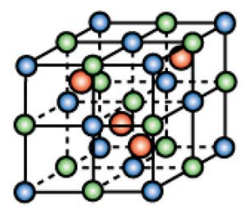

$\mathrm{Cl}_{\mathrm{b}}$ structure

(atomic radius, atomic mass)

O: $4 c$ site $\left(r_{1}, m_{1}\right)$

O: $4 a$ site $\left(r_{2}, m_{2}\right)$

O: $4 b$ site $\left(r_{3}, m_{3}\right)$

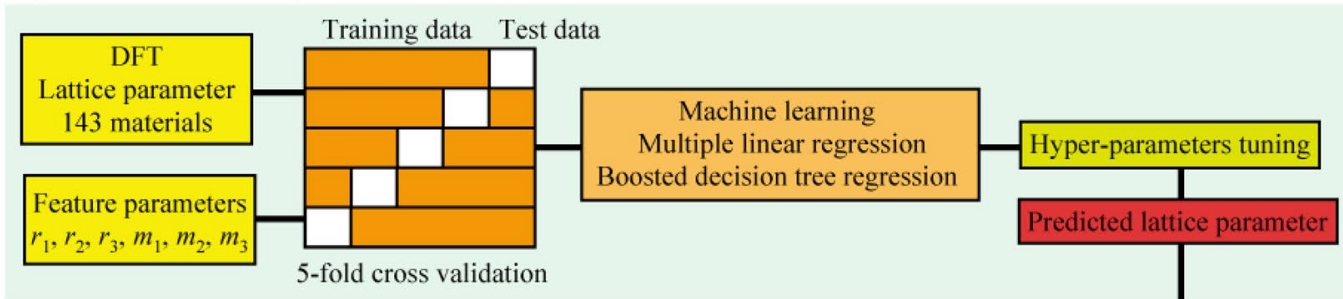

(b) Prediction of thermal conductivity

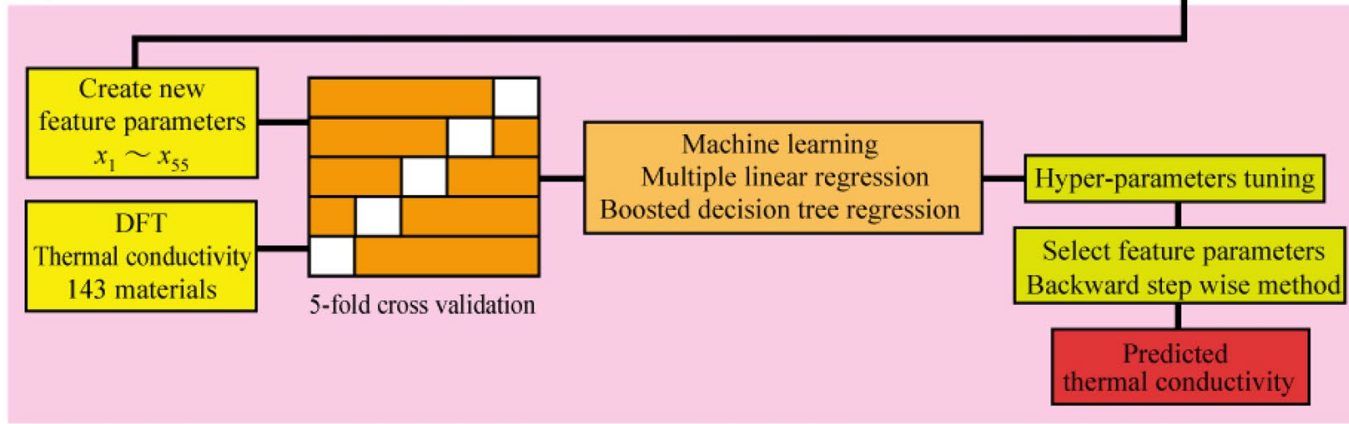

Figure 1. Flowchart of the ML used to (a) prediction of lattice parameter and (b) prediction of thermal conductivity.

compounds ${ }^{12,23-26}$. Therefore, it is difficult to perform comprehensive lattice thermal conductivity calculations for a large number of materials.

Cubic half-Heusler compounds have drawn significant attention in various fields as candidate materials for thermoelectric energy conversion ${ }^{27-35}$ and spintronics technology $y^{36-40}$. This is because it has various electronic structures, such as semi-metals, semiconductors, and a topological insulator. Carrete et al. ${ }^{41}$ and Liu et al. ${ }^{42}$ attempted to predict the lattice thermal conductivity of half-Heusler compounds obtained from the results of the DFT calculations by ML. Carrete et al. reported that the lattice thermal conductivity of half-Heusler compounds can be predicted in the range of $10 \%$ using the Young's modulus value obtained from the DFT calculations as the descriptor for the ML. Liu et al. reported that the lattice thermal conductivity of half-Heusler compounds can be predicted in the range of $10 \%$ using the atomic numbers, atomic masses, and atomic radii of the constituent atoms of half-Heusler compounds as the descriptors for ML.

The prediction of the lattice thermal conductivity must be highly accurate to predict the performance of thermoelectric materials and thermal management of electronic devices. Therefore, we have developed a ML algorithm to predict the lattice parameter and lattice thermal conductivity of half-Heusler compounds from the atomic information of their constituent atoms (atomic radius and atomic mass) only. This algorithm can predict lattice thermal conductivity values with high accuracy of less than $4 \%$ for many half-Heusler compounds. In this study, we found that the lattice thermal conductivity of the half-Heusler compounds, which is difficult to predict using the DFT calculations, can be predicted with high accuracy by ML. This report will provide a breakthrough in the development of new materials, as it can contribute to the discovery of innovative materials for next-generation thermoelectric conversion and spintronics materials, and other technologies requiring thermal management in the future.

\section{Results and discussions}

Figure 1 shows a flowchart of the ML used to predict the lattice parameter and lattice thermal conductivity in half-Heusler compounds. The atomic radii, $r_{1}, r_{2}, r_{3}$, and atomic masses, $m_{1}, m_{2}, m_{3}$, of the elements at $4 c, 4 a$, and $4 b$ sites in the $\mathrm{Cl}_{\mathrm{b}}$-type crystal structure were used as descriptors. The Python library Pymatgen, Python Materials Genomics $^{43}$, was used to obtain the elemental information. The swapped data set of the elemental information of the $4 a$ and $4 b$ sites was also created because the $4 a$ and $4 b$ sites are interchangeable. First, to build a ML model to predict the lattice parameters, the atomic radii and masses were used as descriptors. Second, to build a ML model to predict the calculated lattice thermal conductivity, the parameters generated by various combinations of atomic masses, atomic radii, and the lattice parameters were used. The parameters of the combinations of atomic radii and atomic masses used for lattice thermal conductivity prediction are listed in Table S2 in the Supplementary materials section. Finally, to find the best combination of parameters, we used the Wrapper method with a backward feature elimination to sequentially remove unimportant parameters hence build an optimal ML model for predicting the lattice thermal conductivity. For lattice parameter and lattice thermal conductivity prediction, the multiple linear regression and boosted decision tree regression models were used as the ML models. The hyperparameters were adjusted by random sweeps to adjust the optimal hyperparameters. Python 3.6 was used for implementing the ML model. The training and test data were split between 80 and $20 \%$. We used fivefold cross validation on the data set to evaluate the decision coefficients using the test data for each fold, and the mean of the decision coefficients, $R^{2}$, was used to evaluate the ML model. 

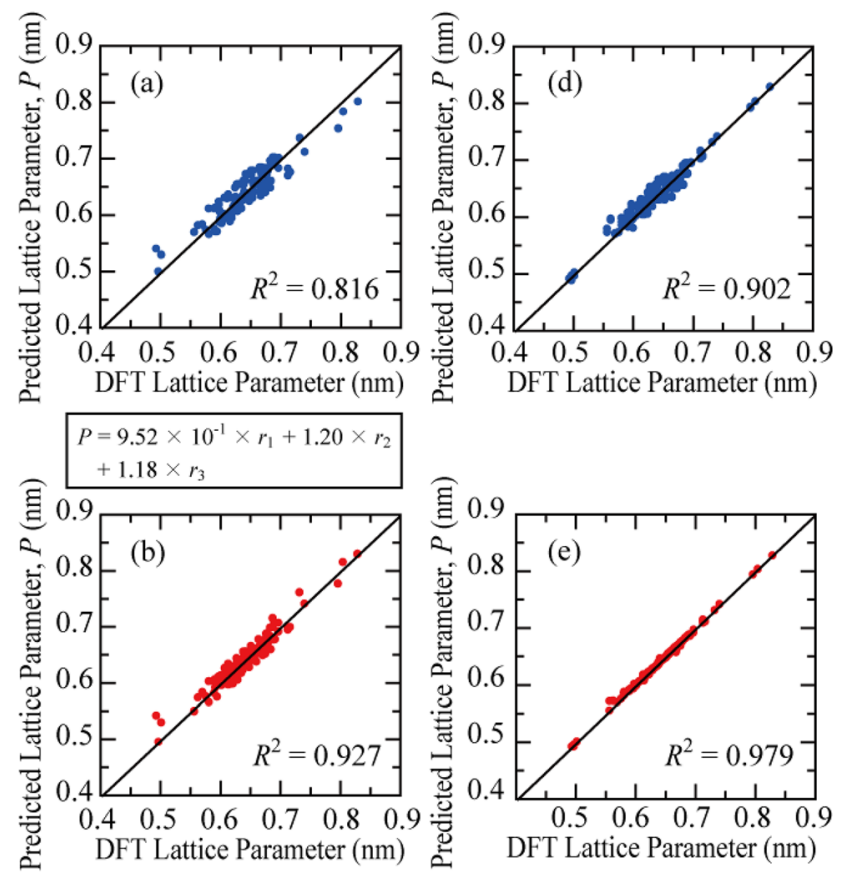

$\begin{aligned} P & =1.62 \times 10^{-3} \times m_{1}-1.21 \times 10^{-3} \times m_{2} \\ & -1.32 \times 10^{-4} \times m_{3}+8.88 \times 10^{-1} \times r_{1} \\ & +1.36 \times r_{2}+1.38 \times 10^{-1} \times r_{3}\end{aligned}$

\begin{tabular}{|l|l|l|l|l|l|l|}
\hline 1 & 1 & 1 & 1 & 1 \\
\hline
\end{tabular}
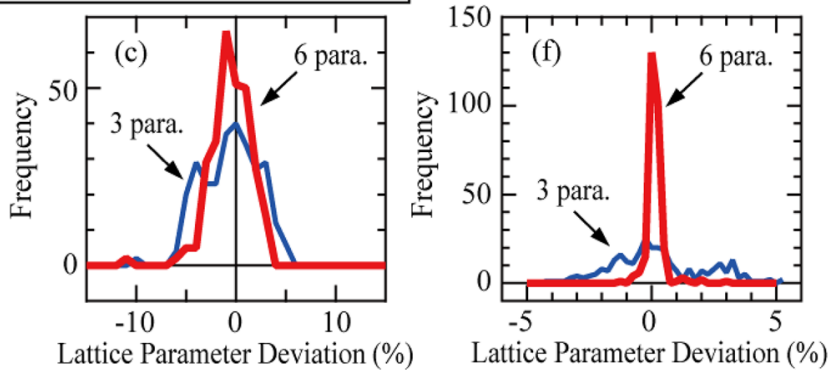

Lattice Parameter Deviation (\%)

Lattice Parameter Deviation (\%)

Figure 2. Comparison between the lattice parameters predicted by DFT calculations and those predicted by: $(\mathbf{a}-\mathbf{c})$ the ML model of multiple linear regression and $(\mathbf{d}-\mathbf{f})$ the ML model of boosted decision tree regression. $(\mathbf{a}, \mathbf{d})$ and $(\mathbf{b}, \mathbf{e})$ are the results of ML using a combination of atomic radii (3 parameters) and atomic mass and atomic radius ( 6 parameters) as descriptors, respectively. The regression equations determined by multiple linear regression are shown at the bottom of figures $(\mathbf{a}, \mathbf{b})$. (c,f) Frequency of deviations between the calculated and predicted lattice parameters.

Figure $2 \mathrm{a}-\mathrm{c}$ show the results of ML using multiple linear regression of the lattice parameters for various halfHeusler compounds obtained from the structural optimization by DFT calculations. ML with multiple linear regression predicts the lattice parameter with a higher accuracy using the atomic masses and atomic radii than that using the atomic radii only. As described in Table S1, the stability of half-Heusler compounds is affected by the atomic radii of each site and the atomic masses. Therefore, besides the atomic radii, the atomic masses have an important influence on the lattice parameter determination. The prediction of the lattice parameter using multiple linear regression can be determined with an accuracy of approximately 5\%, as shown in Fig. $2 c$. To determine the lattice parameter with a better accuracy, we performed ML by boosted decision tree regression as shown in Fig. 2d-f. Using atomic radius and mass as parameters, $R^{2}$ improved to 0.979 . As shown in Fig. $2 \mathrm{f}$, the ML model reproduced the lattice parameter almost perfectly with an accuracy of approximately $\pm 1 \%$. The boosted decision tree regression using the atomic radii and masses of the atoms at the three sites of the $\mathrm{Cl}_{\mathrm{b}}$-type structure as a description was found to be the most suitable for predicting the lattice parameter by ML.

Figure 3 show the results of the predicted lattice thermal conductivity for various half-Heusler compounds using ML with multiple linear regression and boosted decision tree regression. As shown in Fig. 3a, the boosted decision tree regression model shows a higher coefficient of determination than the multiple linear regression model, and reproduces the thermal conductivity of the half-Heusler compounds well. The ML model, such as a simple multiple linear regression is not suitable for ML of the lattice thermal conductivities. This result suggests that the lattice thermal conductivity exhibits a high accuracy owing to the complex interaction of various descriptors. Figure $3 \mathrm{~b}$ shows the feature importance scores for the 55 parameters in ML of boosted decision tree regression. Among the 55 parameters, the top 4 parameters make a significant contribution to the accuracy 

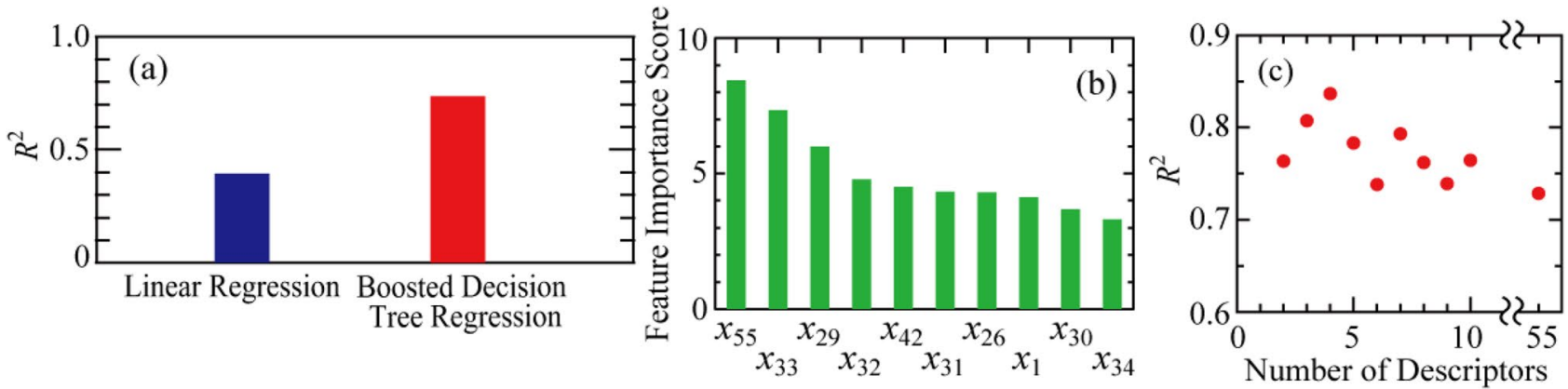

Figure 3. (a) $R^{2}$ for the ML model of multiple linear regression and boosted decision tree regression performed on the calculated lattice thermal conductivity. (b) Top 10 parameters of permutation feature importance for each parameter in the ML model of boosted decision tree regression. (c) Dependence of the number of features on the $R^{2}$ evaluated by the wrapper Method with backward feature elimination.
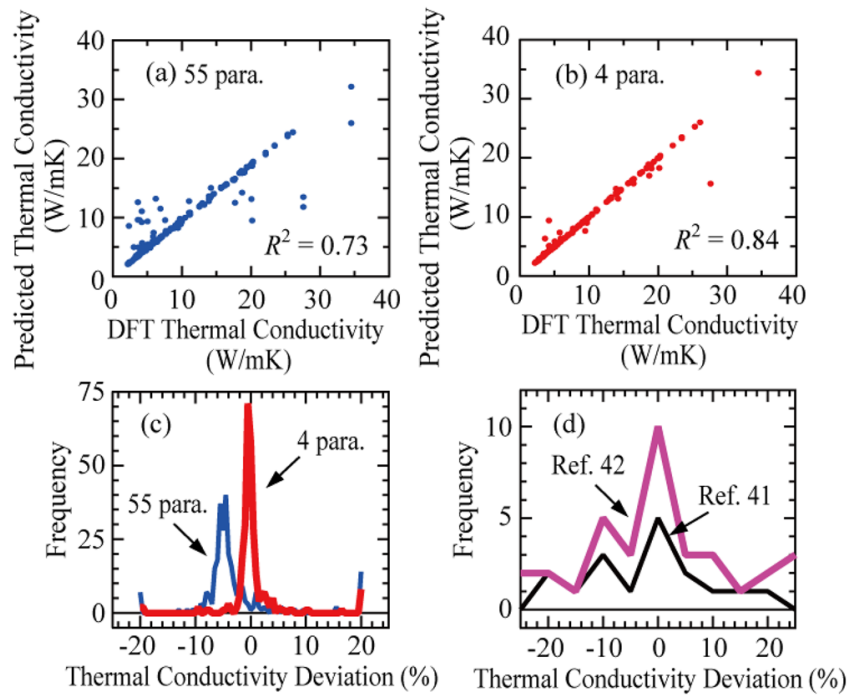

Figure 4. (a,b) Comparison between the lattice thermal conductivity predicted by the ML model of boosted decision tree regression using 55, and 4 parameters and the lattice thermal conductivity calculated by the DFT calculation. (c) Number of deviations between the predicted and calculated lattice thermal conductivity using 55 and 4 parameters. (d) Number of deviations between the predicted and calculated lattice thermal conductivity reported by Carrete et al. ${ }^{41}$ and Liu et al. ${ }^{42}$.

of ML. It is known that when many 55 parameters are used in ML, the prediction accuracy is reduced because of over-fitting. It is necessary to find the best combination of parameters to improve the prediction accuracy of the lattice thermal conductivity. Figure $3 \mathrm{c}$ shows the results of the evaluation by the Wrapper Method using backward feature elimination. The backward feature elimination calculates the permutation feature importance of each parameter and sequentially removes the unimportant parameter to find the optimal combination of parameters. The $R^{2}$ for the ML of thermal conductivity using the top four parameters is the highest $R^{2}$ of 0.84 , which is an improvement over the $R^{2}$ when all the parameters are considered. The best parameter combination of the important features score for the prediction of the lattice thermal conductivity are in the following order:

(Top 1) $x_{55}$ : lattice parameter.

(Top 2) $x_{42}$ : the difference between the mean atomic radius of the constituent elements and the atomic radius of the $4 c$ site, $\left(r_{1}+r_{2}+r_{3}\right) / 3-r_{1}$.

(Top 3) $x_{33}$ : the difference between the mean atomic mass of the constituent elements and the atomic mass of the $4 c$ site, $\left(m_{1}+m_{2}+m_{3}\right) / 3-m_{1}$.

(Top 4) $x_{29}$ : the sum of the atomic masses, $m_{1}+m_{2}+m_{3}$.

Figure 4a,b show the results of lattice thermal conductivity predicted by ML for the boosted decision tree regression described with 55 and 4 parameters, respectively. The predictions of thermal conductivity with 4 parameters are in a better agreement with the results of ML with 55 parameters. Figure $4 \mathrm{c}$ shows the number of deviations between the predicted and calculated lattice thermal conductivities by the ML model of the boosted decision tree regression with 55 and 4 parameters. When 55 parameters were used, the lattice thermal 


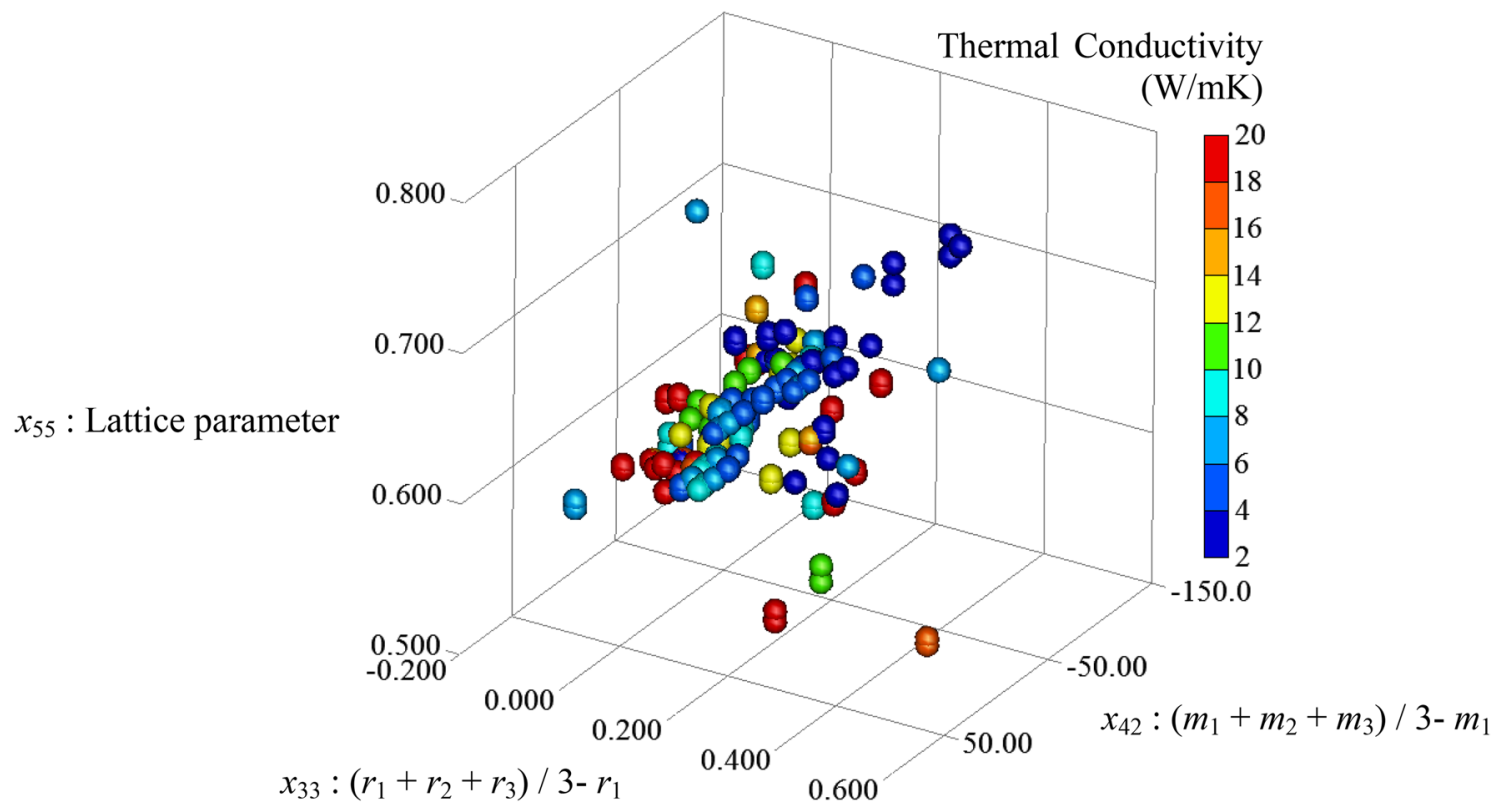

Figure 5. 3D plots of the three parameters $\left(x_{33}, x_{42}, x_{55}\right)$ and predicted lattice thermal conductivity. The magnitude of the lattice thermal conductivity in $3 \mathrm{D}$ plotting is shown in color scale.

conductivity was overestimated, with a deviation of approximately $8 \%$. Conversely, when 4 parameters were used to describe the lattice thermal conductivity, the accuracy improved to approximately $\pm 4 \%$. Carrete et al. ${ }^{41}$ and Liu et al. ${ }^{42}$ have previously reported that the accuracy of the lattice thermal conductivity prediction in half-Heusler compounds predicted by ML is approximately $\pm 10 \%$, as shown in Fig. $4 \mathrm{~d}$. In this study, our ML model using the lattice parameter as a descriptor and selecting an appropriate combination of atomic radii and atomic masses as a descriptor led to a significant improvement in the accuracy of the prediction of lattice thermal conductivity.

Figure 5 plots the relationship between three parameters and thermal conductivity, which are important in determining the lattice thermal conductivity of half-Heusler compounds. For many compounds with large lattice parameters, the lattice thermal conductivity is below 6 , which is a low lattice thermal conductivity material. The lattice thermal conductivity of a solid material is expressed by the equation $\kappa \sim C v l$, where $C$, $v$, and $l$ represent the specific heat, sound velocity, and phonon mean free path, respectively. It is essential to reduce the thermal conductivity to improve the performance of thermoelectric conversion materials. For this purpose, $C$, $v$, and $l$ should be reduced. However, it is difficult to change $C$ and $l$ significantly in the same crystal system. The lattice thermal conductivity is lower in a compound with a large sum of atomic mass, including the structure and small lattice parameter because the sound velocity is expressed as a function inversely proportional to the density. Therefore, for compounds with small lattice parameters, the decrease in thermal conductivity can be qualitatively explained by the decrease in sound velocity. In half-Heusler compounds with lattice parameters of 0.7 to 0.8 , the thermal conductivity is below 4 for the systems BaNaSb, KBaSb, CaCdSn, and KSrSb with large differences between the average atomic mass and $4 c$ sites in the compounds. Even in half-Heusler compounds with large lattice parameters, small lattice thermal conductivities can be achieved by selecting the heavy elements to occupy the $4 c$ site.

The material design of half-Heusler compounds has focused on compounds with elements from group 1 to 4 applied to the $4 a$ site, elements from group 12 to 16 applied to the $4 b$ site, and elements from group 8 to 11 applied to the $4 c$ site. This study revealed that many half-Heusler compounds contain the group 14-16 elements to the $4 c$ site and group 1 to 2 elements to the $4 a$ and $4 b$ sites. When heavier elements, such as Sn and Sb, are occupied to the $4 c$ site, the difference between the average atomic weight in the compound and the $4 c$ site is particularly large, and compounds are predicted to actually show a significant decrease in thermal conductivity. In the future, the synthesis of these groups of materials will lead to new low thermal conductivity half-Heusler compounds.

The computational cost is defined as the computation time required to perform a calculation, considering the number of CPUs used to accomplish the computational process. A computation time of only $0.5 \mathrm{~h}$ was required to develop the prediction model of thermal conductivity using ML, whereas the corresponding DFT calculations for a single compound required approximately $72 \mathrm{~h}$. Therefore, the computation time required to build a model for predicting thermal conductivity using ML is sufficiently smaller than that required for the DFT calculations. This implies that the ML-based prediction models are more efficient than the DFT method for thermal conductivity calculations. Thus, ML-based modeling is a highly effective tool for predicting the thermal conductivity of half-Heusler compounds.

\section{Conclusion}

In this study, we investigated whether the lattice thermal conductivity of half-Heusler compounds can be predicted by ML from the atomic radii and masses of the constituent elements. The results show that the lattice thermal conductivity of the half-Heusler compounds can be predicted with an accuracy of $\pm 4 \%$ using the predicted 
lattice parameters and the atomic radii and masses. In addition to the conventional material design in which a material with a small lattice parameter and low density has a low thermal conductivity, it was found that even for materials with a large lattice parameter, one can design a material with a low thermal conductivity by selecting elements occupying the $4 c$ site of the half-Heusler structure with a larger atomic mass than those occupying the $4 a$ and $4 b$ sites. Using the results of $\mathrm{ML}$, the thermal conductivity of unknown half-Heusler compounds can be instantly predicted. It is expected that the search for half-Heusler compounds with the desired thermal conductivity will become easier and the development of functional materials in a short time and at a low cost will be advanced in the future.

\section{Calculation methods}

Evaluation of site selection of the half-Heusler structure and lattice parameter. Candidate halfHeusler compounds for lattice thermal conductivity calculations were sought from the Materials Project ${ }^{44}$. In the crystal structure of $\mathrm{Cl}_{\mathrm{b}}$-type half-Heusler compounds, there are three types of atomic sites: $4 a(0,0,0), 4 b(0.5$, $0,0)$, and $4 c(0.25,0.25,0.25)$ sites. The $4 a$ and $4 b$ sites are crystallographically interchangeable. Three structural models can be considered as one of the three constituent elements occupies the $4 c$ site and the other two elements occupy the $4 \mathrm{a}$ and $4 \mathrm{~b}$ sites. The lattice parameters were optimized using the DFT calculations for the three models. By comparing the total energies of the three models, the structural model with the lowest energy was determined to be the most stable structural model for the half-Heusler compound. The DFT calculations were performed using the Vienna ab initio Simulation Package (VASP) ${ }^{45-47}$. We adopted the projector augmentedwave (PAW) method ${ }^{48,49}$ with the generalized gradient approximation of Perdew, Burke, and Ernzerh ${ }^{50}$ for the exchange-correlation interactions. The $k$-mesh, cut-off energy and convergence energy were set to $7 \times 7 \times 7$, $600 \mathrm{eV}$, and $10^{-6} \mathrm{eV}$, respectively.

Evaluation of lattice thermal conductivity of half-Heusler compounds. The lattice thermal conductivity of half-Heusler compounds was calculated using the Phono3py code, developed by Atushi Togo et al. ${ }^{22}$. The interatomic force constants were determined using various positions of the atoms in a supercell made of $2 \times 2 \times 2$ primitive cells. Although the lattice thermal conductivity shows a temperature dependence, herein, we discuss the lattice thermal conductivity at $300 \mathrm{~K}$. The calculated lattice thermal conductivities and lattice parameters are summarized in Table S1 in the Supplementary materials section.

Received: 12 March 2021; Accepted: 3 June 2021

Published online: 28 June 2021

\section{References}

1. Müller-Plathe, F. A simple nonequilibrium molecular dynamics method for calculating the thermal conductivity. J. Chem. Phys. 106, 6082 (1997)

2. Volz, S. G. Molecular dynamics simulation of thermal conductivity of silicon nanowires. Appl. Phys. Lett. 75, 2056 (1999).

3. Volz, S. G. \& Chen, G. Molecular-dynamics simulation of thermal conductivity of silicon crystals. Phys. Rev. B 61, 2651 (2000).

4. Turney, J. E., Landry, E. S., McGaughey, A. J. H. \& Amon, C. H. Predicting phonon properties and thermal conductivity from anharmonic lattice dynamics calculations and molecular dynamics simulations. Phys. Rev. B 79, 064301 (2009).

5. Lampina, E., Palla, P. L., Francioso, P.-A. \& Cleri, F. Thermal conductivity of silicon using reverse non-equilibrium molecular dynamics. J. Appl. Phys. 114, 033525 (2013).

6. Tanaka, K., Ogata, S., Kobayashi, R., Tamura, T. \& Kouno, T. A molecular dynamics study on thermal conductivity of thin epoxy polymer sandwiched between alumina fillers in heat-dissipation composite material. Int. J. Heat Mass Transf. 89, 714 (2015).

7. El-Genk, M. S., Talaat, K. \& Cowen, B. J. Thermal conductivity of silicon using reverse non-equilibrium molecular dynamics. J. Appl. Phys. 123, 205104 (2018).

8. Broido, D. A., Malorny, M., Birner, G., Mingo, N. \& Stewart, D. A. Intrinsic lattice thermal conductivity of semiconductors from first principles. Appl. Phys. Lett. 91, 231922 (2007).

9. Ward, A. \& Broido, D. A. Intrinsic lattice thermal conductivity of Si/Ge and GaAs/AlAs superlattices. Phys. Rev. B 77, 245328 (2008).

10. Ward, A. \& Broido, D. A. Intrinsic phonon relaxation times from first-principles studies of the thermal conductivities of Si and Ge. Phys. Rev. B 81, 085205 (2010).

11. Esfarjani, K., Chen, G. \& Stokes, H. T. Heat transport in silicon from first-principles calculations. Phys. Rev. B 84, 085204 (2011).

12. Shiomi, J., Esfarjani, K. \& Chen, G. Thermal conductivity of half-Heusler compounds from first-principles calculations. Phys. Rev. B 84, 104302 (2011).

13. Tian, Z. et al. Phonon conduction in PbSe, $\mathrm{PbTe}$, and $\mathrm{PbTe}_{1-x} \mathrm{Se}_{x}$ from first-principles calculations. Phys. Rev. B 85, 184303 (2012).

14. Shiga, T. et al. Microscopic mechanism of low thermal conductivity in lead telluride. Phys. Rev. B 85, 155203 (2012).

15. Lindsay, L., Broido, D. A. \& Reinecke, T. L. Thermal conductivity and large isotope effect in GaN from first principles. Phys. Rev. Lett. 109, 095901 (2012).

16. Chaput, L. Direct solution to the linearized phonon Boltzmann equation. Phys. Rev. Lett. 110, 265506 (2013).

17. Wan, X. et al. Materials discovery and properties prediction in thermal transport via materials informatics: a mini review. Nano Lett. 19, 3387 (2019).

18. Ouyang, Y. et al. Accuracy of machine learning potential for predictions of multiple-target physical properties. Chin. Phys. Lett. 37, $126301(2020)$.

19. Ouyang, Y., Yu, C., Yan, G. \& Chen, J. Machine learning approach for the prediction and optimization of thermal transport properties. Front. Phys. 16, 43200 (2021).

20. Wang, Y. et al. Prediction of tubular solar still performance by machine learning integrated with Bayesian optimization algorithm. Appl. Therm. Eng. 184, 116233 (2021).

21. Wan, W., Ma, D., Pan, D., Yang, L. \& Yang, N. Optimizing thermal transport in graphene nanoribbon based on phonon resonance hybridization. arXiv:2104.01878 (2021).

22. Togo, A., Chaput, L. \& Tanaka, I. Distributions of phonon lifetimes in Brillouin zones. Phys. Rev. B 91, 094306 (2015). 
23. Ding, G., Gao, G. Y. \& Yao, K. L. Examining the thermal conductivity of the half-Heusler alloy TiNiSn by first-principles calculations. J. Phys. D Appl. Phys. 48, 235302 (2015).

24. Hermet, P. \& Jund, P. Lattice thermal conductivity of NiTiSn half-Heusler thermoelectric materials from first-principles calculations. J. Alloys Compd. 668, 248 (2016).

25. Eliassen, S. N. H. et al. Lattice thermal conductivity of $\mathrm{Ti}_{x} \mathrm{Zr}_{y} \mathrm{Hf}_{1-x-y} \mathrm{NiSn}$ half-Heusler alloys calculated from first principles: key role of nature of phonon modes. Phys. Rev. B 95, 045202 (2017).

26. Han, S. H. et al. High thermoelectric performance of half-Heusler compound BiBaK with intrinsically low lattice thermal conductivity. J. Phys. Condens. Matter 32, 425704 (2020).

27. Aliev, F. G., Kozyrkov, V. V., Moschchalkov, V. V., Scolozdra, R. V. \& Durczewski, K. Narrow band in the intermetallic compounds MNiSn (M = Ti, Zr, Hf). Z. Phys. B 80, 353 (1990).

28. Aliev, F. G. Gap at Fermi level in some new d-and f- electron intermetallic compounds. Phys. B 171, 191 (1991).

29. Kawaharada, Y., Uneda, H., Muta, H., Kurosaki, K. \& Yamanaka, S. High temperature thermoelectric properties of NiZrSn halfHeusler compounds. J. Alloys Compd. 364, 59 (2004).

30. Sakurada, S. \& Shutoh, N. Effect of Ti substitution on the thermoelectric properties of ( $\mathrm{Zr}, \mathrm{Hf}) \mathrm{NiSn}$ half-Heusler compounds. Appl. Phys. Lett. 86, 082105 (2005).

31. Zhu, T., Fu, C., Xie, H., Liu, Y. \& Zhao, X. High efficiency half-Heusler thermoelectric materials for energy harvesting. Adv. Energy Mater. 5, 1500588 (2015).

32. Rausch, E. et al. Fine tuning of thermoelectric performance in phase-separated half-Heusler compounds. J. Mater. Chem. C 3, 10409-10414 (2015).

33. Chai, Y., Oniki, T., Kenjo, T. \& Kimura, Y. The effect of an isoelectronic Ti-Zr substitution on Heusler nanoprecipitation and the thermoelectric properties of a $\left(\mathrm{Ti}_{0.2}, \mathrm{Zr}_{0.8}\right) \mathrm{Ni}_{1.1}$ Sn half-Heusler alloy. J. Alloys Compd. 662, 566 (2016).

34. Tang, Y. et al. Impact of Ni content on the thermoelectric properties of half-Heusler TiNiSn. Energy Environ. Sci. 11, 311 (2018).

35. Miyazaki, H. et al. Probing local distortion around structural defects in half-Heusler thermoelectric NiZrSn alloy. Sci. Rep. 10, 19820 (2020).

36. de Groot, R. A., Mueller, F. M., van Engen, P. G. \& Buschow, K. H. J. New class of materials: half-metallic ferromagnets. Phys. Rev. Lett. 50, 2024 (1983).

37. Feng, L., Liu, E. K., Zhang, W. X., Wang, W. H. \& Wu, G. H. Half-metallic ferromagnetism in new half-Heusler compounds: an ab initio study of CrTiX (X = Si, Ge, Sn, Pb). J. Magn. Magn. Mater. 351, 92 (2014).

38. Zhang, R. L. et al. Two prospective Li-based half-Heusler alloys for spintronic applications based on structural stability and spinorbit effect. J. Appl. Phys. 122, 013901 (2017).

39. Ma, J. et al. Computational investigation of half-Heusler compounds for spintronics applications. Phys. Rev. B 95, 024411 (2017).

40. Dehghan, A. \& Davatolhagh, S. $d^{0}-d$ half-Heusler alloys: a potential class of advanced spintronic materials. J. Alloys Compd. 772, $132(2019)$

41. Carrete, J., Li, W., Mingo, N., Wang, S. \& Curtarolo, S. Finding unprecedentedly low-thermal-conductivity half-Heusler semiconductors via high-throughput materials modeling. Phys. Rev. X 4, 011019 (2014).

42. Liu, J. et al. A high-throughput descriptor for prediction of lattice thermal conductivity of half-Heusler compounds. J. Phys. D Appl. Phys. 53, $315301(2020)$.

43. Ong, S. P. et al. Python Materials Genomics (pymatgen): a robust, open-source python library for materials analysis. Comput. Mater. 68, 314 (2013).

44. Jain, A. et al. The Materials Project: a materials genome approach to accelerating materials innovation. APL Mater. 1, 011002 (2013).

45. Kresse, G. \& Hafner, J. Ab initio molecular dynamics for open-shell transition metals. Phys. Rev. B 48, 13115 (1993).

46. Kresse, G. \& Furthmuller, J. Efficiency of ab-initio total energy calculations for metals and semiconductors using a plane-wave basis set. J. Comput. Mater. Sci. 6, 15 (1996).

47. Kresse, G. \& Furthmuller, J. Efficient iterative schemes for ab initio total-energy calculations using a plane-wave basis set. Phys. Rev. B 54, 11169 (1996).

48. Blochl, P. E. Projector augmented-wave method. Phys. Rev. B 50, 17953 (1994)

49. Kresse, G. \& Joubert, D. From ultrasoft pseudopotentials to the projector augmented-wave method. Phys. Rev. B 59, 1758 (1999).

50. Perdew, J. P., Burke, K. \& Ernzerhof, M. Generalized gradient approximation made simple. Phys. Rev. Lett. 77, 3865 (1996).

\section{Acknowledgements}

The computations were performed by the Research Center for Computational Science, Okazaki, Japan. This study was partly supported by the Japan Society for the Promotion of Science's (JSPS) Grants-in-Aid for Scientific Research (KAKENHI) (C) (17K06771, 18K04700, 18K04748, 20K05100, 20K05060). We would like to thank Editage (www.editage.com) for English language editing.

\section{Author contributions}

H.M. performed DFT calculations. H. M. and T. T. performed ML calculations. H. M wrote the manuscript. Y.N supervised this study. All the authors discussed the results and commented on the manuscript.

\section{Competing interests}

The authors declare no competing interests.

\section{Additional information}

Supplementary Information The online version contains supplementary material available at https://doi.org/ 10.1038/s41598-021-92030-4.

Correspondence and requests for materials should be addressed to H.M.

Reprints and permissions information is available at www.nature.com/reprints.

Publisher's note Springer Nature remains neutral with regard to jurisdictional claims in published maps and institutional affiliations. 
(c) (i) Open Access This article is licensed under a Creative Commons Attribution 4.0 International cc) License, which permits use, sharing, adaptation, distribution and reproduction in any medium or format, as long as you give appropriate credit to the original author(s) and the source, provide a link to the Creative Commons licence, and indicate if changes were made. The images or other third party material in this article are included in the article's Creative Commons licence, unless indicated otherwise in a credit line to the material. If material is not included in the article's Creative Commons licence and your intended use is not permitted by statutory regulation or exceeds the permitted use, you will need to obtain permission directly from the copyright holder. To view a copy of this licence, visit http://creativecommons.org/licenses/by/4.0/.

(C) The Author(s) 2021 